\title{
Enzyme Linked Immunosorbent Assays Based on Recombinant S1 and Its Truncated Proteins to Measure Porcine Serum IgA Antibody Against PEDV Could Reflect Neutralization Activity
}

\section{Ying Shan}

Zhejiang Provincial Key Lab of Preventive Veterinary Medicine, MOA Key Laboratory of Animal Virology, Center for Veterinary Sciences, Zhejiang University

\section{Qin Gao}

Department of Veterinary Medicine, College of Animal Sciences, Zhejiang University Junyong Mao

Hainan Institute, Zhejiang University

Jingyou Zheng

Department of Veterinary Medicine, College of Animal Sciences, Zhejiang University

\section{Xiaohan Xu}

Department of Veterinary Medicine, College of Animal Sciences, Zhejiang University

\section{Chuni Zhang}

Department of Veterinary Medicine, College of Animal Sciences, Zhejiang University

\section{Xiaojun Huang}

Department of Veterinary Medicine, College of Animal Sciences, Zhejiang University

\section{Jidong Xu}

Department of Veterinary Medicine, College of Animal Sciences, Zhejiang University

\section{Fushan Shi}

Zhejiang Provincial Key Lab of Preventive Veterinary Medicine, MOA Key Laboratory of Animal Virology, Center for Veterinary Sciences, Zhejiang University

\section{Min Yue}

Zhejiang Provincial Key Lab of Preventive Veterinary Medicine, MOA Key Laboratory of Animal Virology, Center for Veterinary Sciences, Zhejiang University

\section{Fang He}

Zhejiang Provincial Key Lab of Preventive Veterinary Medicine, MOA Key Laboratory of Animal Virology, Center for Veterinary Sciences, Zhejiang University

\section{Weihuan Fang}

Department of Veterinary Medicine, College of Animal Sciences, Zhejiang University

Xiaoliang Li ( $\nabla$ xlli@zju.edu.cn ) 
Zhejiang Provincial Key Lab of Preventive Veterinary Medicine, MOA Key Laboratory of Animal Virology, Center for Veterinary Sciences, Zhejiang University

\section{Research Article}

Keywords: PEDV, Bac-to-Bac Eukaryotic expression, recombinant S1 truncated protein, ELISA

Posted Date: February 25th, 2022

DOI: https://doi.org/10.21203/rs.3.rs-1312284/v1

License: (a) (i) This work is licensed under a Creative Commons Attribution 4.0 International License. Read Full License 


\section{Abstract}

Porcine epidemic diarrhea virus (PEDV) can infect pigs of all ages, especially piglets. PEDV has spread across Asia since the 1980s. The highly virulent variant PEDV broke out on a large scale and caused huge economic losses to the pig industry in late 2010 in China. Rapid methods with high specificity are urgently needed for the diagnosis and control of the disease. In a previous study, we successfully established an ELISA method to detect porcine serum IgG antibody against PEDV based on full-length PEDV S1 protein as a coating antigen. In this study, the correlation between serum neutralizing activity and the value of IgA ELISA in porcine serum was further explored. First, we divided the PEDV S1 gene into three segments and constructed the recombinant plasmids pFastBac1-S1T1 (aa 21-279), pFastBac1S1T2 (aa 280-539) and pFastBac1-S1T3 (aa 540-788), which carry the different antigenic regions of the S1 gene. Truncated S1 proteins PEDV-S1T1/S1T2/S1T3 were obtained by a Bac-to-Bac expression system, with protein sizes of $36 \mathrm{kDa}, 38 \mathrm{kDa}$ and $38 \mathrm{kDa}$, respectively. Recombinant proteins presented high reactivity with the monoclonal antibody against PEDV and positive pig serum. A total of 213 clinical serum samples were tested by the above indirect ELISA methods, and IFA was used as the gold standard. ROC curves revealed a significant correlation between S1-ELISA and S1T2-ELISA with a 0.9134 correlation coefficient and favourable sensitivity and specificity of S1-ELISA (93.24\%, 95.68\%) and S1T2ELISA $(89.33 \%, 94.16 \%)$. Our results also indicated that serum with higher neutralizing activity had a higher IgA titer based on S1-ELISA, S1T1-ELISA and S1T2-ELISA. In conclusion, both S1-ELISA and S1T2ELISA can be used as candidate systems for detecting anti-PEDV IgA antibody titers in serum, which can reflect the level of neutralizing activity in pigs after natural infection or vaccination. The above research results provide a basis for the prevention and control of PEDV and can be used in the detection of host anti-infective immunity and evaluation of vaccine immune effects.

\section{Introduction}

Porcine epidemic diarrhea (PED) first appeared in the United Kingdom[1] and Belgium[2] in the early 1970s and then gradually spread to other European countries. After the 1990s, the disease showed sporadic local outbreaks in Europe[3]. Porcine epidemic diarrhea virus (PEDV) has gradually become popular in Asian countries since it was first reported in Asia in 1982. In October 2010, the highly virulent variant PEDV broke out on a large scale and then spread across the country in China[4]. Survey data showed that the detection rates of PEDV-positive samples ranged from $61.10-78.49 \%$, while the rates of PEDV-positive pig farms ranged from $71.43-83.47 \%[5,6]$. Pigs of all ages are affected by PEDV, among which the mortality rate of suckling piglets is as high as $100 \%[4,7,8]$. The high morbidity and mortality have brought huge economic losses to the pig industry.

PEDV is a member of the alpha coronavirus family with a particle approximately 95-190 nm in diameter[1] and has an envelope, a genome length of approximately $28 \mathrm{~kb}$ containing the $5^{\prime}$ noncoding region (5'-UTR), a single-stranded positive-stranded RNA and a cap structure and a poly A tail in the $3^{\prime}$-end noncoding region (3'-UTR) [2]. The viral genome consists of a 5'-UTR, 3'-UTR and seven open reading frames (ORF1a, ORF1b, ORF2, ORF3, ORF4, ORF5 and ORF6). The replicase polyprotein 1ab (pp1ab) 
encoded by ORF1a and ORF1b is cleaved by papain (PL pro) and 3C-like protease (3CL pro) into 16 nonstructural proteins (Nsp1-16) [9, 10]; ORF2-6 encode ORF3 nonstructural proteins and four structural proteins, including spike protein (S protein), small membrane protein (E protein), membrane glycoprotein (M protein) and nucleocapsid protein (N protein) [11].

Sequence analysis of the whole PEDV genome showed that its hypervariable regions were mainly concentrated in the nsp2 gene[12], nsp3 gene[12], S gene[13], and ORF3 gene[14]. Among them, the S gene was the most highly mutagenic. The PEDV S protein is composed of 1383 amino acids with a size of approximately $180-200 \mathrm{kDa}$. It is located on the surface of the coronavirus and contains a signal peptide, a neutralizing epitope, a transmembrane domain and a short intracellular region. The $S$ gene of some coronaviruses contains a furin cleavage site, which can be cleaved into two subunits, S1 and S2[15], but the PEDV S protein cannot be naturally recognized due to the lack of this endoprotease recognition site. The PEDV S protein is divided into two domains according to its function: the N-terminal S1 subunit (aa 1-789) is responsible for recognizing cell surface receptors, and the C-terminal S2 subunit (aa 790-1383) is responsible for mediating virus and cell membrane fusion. When the virus invades the cell, the S1 protein binds to the surface of the host cell and induces the production of neutralizing antibodies; the S2 protein mediates the fusion of the virus with the host cell membrane, allowing the virus genome to enter the host cell. As the most mutagenic protein in PEDV, the $S$ protein can change the pathogenicity and tissue tropism of the virus by gene insertion, deletion and mutation[4], so it is often used in epidemiological investigations.

Antibody detection tests can be used to evaluate the immune effect of vaccines and monitor the disease status. The current methods for detecting PEDV antibodies mainly include indirect immunofluorescence (IFA), serum neutralization tests (SVNs), enzyme-linked immunosorbent assays (ELISAs) and fluorescent microsphere immunoassays (FMIAs). Indirect immunofluorescence (IFA) is based on the antigenantibody reaction and evaluates the immune status of the population by specifically detecting PEDV antibodies in the serum of suspicious populations [16]. Zhu Weizheng and others collected pig serum samples at different time points after recovery, and the positive rate was approximately $89.25 \%$ by IFA. Song et al. [17] verified the reliability of IFA as the gold standard for serological testing by analyzing the correlation between IFA and ELISA[18]. ELISA detection methods for PEDV antibodies mainly include indirect ELISA and blocking ELISA [19-21]. Wager et al. first used indirect ELISA to detect IgG antibodies in human serum albumin in 1978[22]. Yorde et al. established a blocking ELISA detection method in 1976. The established ELISA detection methods for PEDV mainly focus on PEDV S protein, $\mathrm{N}$ protein and $\mathrm{M}$ protein. M. Knuchel et al. extracted viral proteins from Vero cells infected with PEDV, adjusted the solubility of the protein in the buffer by changing the $\mathrm{pH}$ value, enriched PEDV N protein and S protein, and established an ELISA detection system as a coating antigen [23]. Studies have found that S-ELISA is more sensitive than N-ELISA, and antibodies against $S$ protein last longer in the body than $\mathrm{N}$ protein [23].

Secretory immunoglobulin A ( $\operatorname{gA} A)$, which can neutralize viruses and prevent viruses from invading the intestinal epithelium, plays an important role in intestinal mucosal immunity[24]. A few ELISA methods could detect IgA antibodies against PEDV and be applied to clinical diagnosis or vaccine effect 
evaluation. However, there is no more information on the correlation between neutralization activity and ELISA detection based on recombinant S1 and its truncated proteins for clinical serum samples. In this study, we compared the sensitivity and specificity of ELISA detection of PEDV IgA with full-length S1 and its truncated proteins as coating antigens via ROC curves and analyzed the correlation between neutralization activity in vitro and PEDV IgA antibody levels measured by ELISA. The anti-PEDV IgA antibody levels detected by S1-ELISA or S1T2-ELISA can reflect the neutralization capability for pig serum.

\section{Methods}

\subsection{Strains and plasmids}

E. coli DH5a and DH10Bac were cultured in Luria broth (LB) medium in a rotatory incubator (150 rpm) at $37^{\circ} \mathrm{C}$. DH5a was used to preserve the positive clones in the process of plasmid construction. DH10Bac was used to generate the recombinant bacmid. The pFastBac ${ }^{\text {TM }}$ vector (Life Technologies, USA) was used as the backbone vector to construct the recombinant expression plasmids.

The PEDV S1 gene was analyzed by the transmembrane area prediction server (http://sbcb.bioch.ox.ac.uk/TM_noj/TM_noj.html) and divided into three segments: S1T1, S1T2 and S1T3. The primers used are listed in Table 1. The three segments were amplified from the PEDV S1 gene fragment, which was modified based on the codon usage bias of the SF9 cell line in a previous study [25]. The truncated $\mathrm{S} 1$ fragments were then fused with melittin signal peptides at the $\mathrm{N}$ terminal and $6 \times \mathrm{His}$ tags at the $C$ terminal. The modified truncated $S 1$ fragments were inserted into the $p F a s t B a c^{T M}$ vector between the EcoRI and HindIII restriction endonuclease sites and named pFastBac-S1T1, pFastBac-S1T2 and pFastBac-S1T3. 
Table 1

primers used in this study

\begin{tabular}{|c|c|c|}
\hline Primer & Nucleotide sequence $\left(5^{\prime}\right.$ to $\left.3^{\prime}\right)$ & $\begin{array}{l}\text { Product Length } \\
\text { (bp) }\end{array}$ \\
\hline $1-\mathrm{F}$ & CCGGAATTCATGAAATTCTTAGTCAACGTTGCCCTTG & 90 \\
\hline $1-\mathrm{R}$ & AGAACCGCTGCCGGAACCC & \\
\hline $\begin{array}{l}\text { 2-S1T1- } \\
\mathrm{F}\end{array}$ & GGGTTCCGGCAGCGGTTCTCAAGATGTGACTCGTTGCTCCGC & 777 \\
\hline $\begin{array}{l}\text { 2-S1T1- } \\
\mathrm{R}\end{array}$ & CAGCAAGGGTTGGTTGCTGACC & \\
\hline $\begin{array}{l}\text { 2-S1T2- } \\
\mathrm{F}\end{array}$ & GGGTTCCGGCAGCGGTTCTGTCAACTGTCTCTTGGCTATCCCTAAGATC & 780 \\
\hline $\begin{array}{l}\text { 2-S1T2- } \\
\mathrm{R}\end{array}$ & CGAGAAGCCGTTGATAGTGGTGTCA & \\
\hline $\begin{array}{l}\text { 2-S1T3- } \\
\mathrm{F}\end{array}$ & GGGTTCCGGCAGCGGTTCTTCCTTCTGTGTCGATACCCGCC & 747 \\
\hline $\begin{array}{l}\text { 2-S1T3- } \\
\mathrm{R}\end{array}$ & TGAGAAGTTGGTCGGGATGGAGAT & \\
\hline $\begin{array}{l}\text { 3-S1T1- } \\
\mathrm{F}\end{array}$ & GGTCAGCAACCAACCCTTGCTGGGTTCCGGCAGCGGTTCTCA & 57 \\
\hline $\begin{array}{l}\text { 3-S1T2- } \\
\mathrm{F}\end{array}$ & TGACACCACTATCAACGGCTTCTCGGGTTCCGGCAGCGGTTCTCA & \\
\hline $\begin{array}{l}\text { 3-S1T3- } \\
\mathrm{F}\end{array}$ & ATCTCCATCCCGACCAACTTCTCAGGTTCCGGCAGCGGTTCTCA & \\
\hline $3-\mathrm{R}$ & CCCAAGCTTTTAGTGATGGTGATGGTGGTGATGATG & \\
\hline
\end{tabular}

\subsection{Cells and virus}

The SF9 insect cell line, cultured in SF900III medium (Lifetechnology, USA) supplemented with antibiotics consisting of $0.1 \mathrm{mg} / \mathrm{mL}$ streptomycin, 100 units $/ \mathrm{mL}$ penicillin, and $0.25 \mu \mathrm{g} / \mathrm{mL}$ amphotericin $B$ at $28^{\circ} \mathrm{C}$ in a rotatory incubator $(120 \mathrm{rpm})$, was used to obtain recombinant $\mathrm{S} 1$ truncated protein. The Vero E6 cell line, cultured in DMEM with the antibiotics mentioned above and $10 \%$ fetal bovine serum (FBS, Gibco, USA), was used for virus propagation.

The PEDV epidemic strain ZJ15XS0101 was described in a previous study [25]. Recombinant baculovirus with high viral titers was generated in recombinant bacmid-transferred SF9 cells after two rounds of amplification.

\subsection{Serum and antibody}


Different positive sera were used to detect the specificity of ELISA. Classical swine fever virus (CSFV)-, porcine circovirus type 2 (PCV2)- and porcine reproductive and respiratory syndrome virus (PRRSV)positive serum samples were prepared and kept by our lab[25]. Transmissible gastroenteritis virus (TGEV)-positive serum was kindly provided by Hunan University. PEDV reference positive and negative sera were generated and kept by our lab[25].

A total of 213 pig blood samples were collected from Aug 2013 to Apr 2019 from local farms in Zhejiang Province. Serum was separated from blood samples by centrifugation at $1500 \times \mathrm{g}$ for $10 \mathrm{~min}$ and kept at $-20^{\circ} \mathrm{C}$ until use.

Previously produced mouse anti-S1 monoclonal antibody 6B4/6G1/1C11 strain, PEDV positive serum[25] and mouse anti-His monoclonal antibody (Beyotime, China) were used as primary antibody. HRP-labeled goat anti-mouse IgG antibody (Beyotime, China). HRP-labeled goat anti-pig IgG antibody(Lifetechnology, USA), Alexa Fluor 488-conjugated goat anti-pig IgA antibody(Lifetechnology, USA) and Alexa Fluor 568conjugated goat anti-mouse IgG antibody (Lifetechnology, USA) were used as secondary antibody.

\subsection{Eukaryotic expression and purification of recombinant S1 truncated protein}

Eukaryotic expression and purification of recombinant protein methods were described in detail in previous study[25]. Briefly, recombinant plasmid containing S1T1, S1T2 and S1T3 were transformed into E. coli DH10Bac. The recombinant bacmid was generated and screened by blue-white selection and verified by PCR. The recombinant bacmid was then transfected into SF9 cells using Lipofectamine 2000 (Invitrogen, USA). We analyzed truncated S1 proteins expression in transfected SF9 cells after transfection. Viral supernatant from positive wells was collected and stored at $-80^{\circ} \mathrm{C}$. The virus was amplified and the virus titer was determined. the truncated S1 recombinant proteins were expressed in the amplified recombinant baculovirus infected SF9 cells $(\mathrm{MOI}=0.1)$. The supernatants containing truncated $\mathrm{S} 1$ proteins were separated from cell cultures at $72 \mathrm{~h}$ post infection. For purification, $200 \mathrm{~mL}$ supernatant was added to Ni-NTA column (Yeasen, China). After washing with $10 \mathrm{mM}$ imidazole, The purified truncated $\mathrm{S} 1$ recombinant protein was eluted with $5 \mathrm{~mL}$ of $400 \mathrm{mM}$ imidazole and stored at $-80^{\circ} \mathrm{C}$.

\subsection{Recombinant S1 truncated protein identification}

Recombinant proteins were identified by immunofluorescence assay in SF9 cells as well as Coomassie Blue staining and western blot analysis of purified proteins.

The immunofluorescence assay methods were described in detail in previous study[25]. Briefly, SF9 cells were fixed at $72 \mathrm{hr}$ after recombinant bacmids transfection in $4 \%$ paraformaldehyde at $4^{\circ} \mathrm{C}$ overnight and then washed with $1 \times$ PBS $(0.01 \mathrm{M} \mathrm{pH} 7.2)$. Fixed cells were permeabilized with $0.2 \%$ Triton $\mathrm{X}-100$ at room temperature for 15 min and blocked with PBS containing 10\% BSA for $1 \mathrm{~h}$. The samples were reacted with mouse anti-His monoclonal antibody (dilution 1:1000) as primary antibodies at $37^{\circ} \mathrm{C}$ for $1 \mathrm{~h}$. Alexa Fluor 568-Goat anti-mouse IgG (dilution 1:1000) was used as the secondary antibody at $37^{\circ} \mathrm{C}$ for $1 \mathrm{~h}$. 
Cell nuclei were stained with DAPI (Beyotime, China) at a 1:1000 dilution. Immunofluorescences were visualized using a laser confocal microscope IX81-FV1000 (Olympus, Japan).

Coomassie Blue staining and western blot analysis methods were described in detail in previous study[25]. Briefly, protein samples were separated by SDS-PAGE, stained with Coomassie Blue for $3 \mathrm{~h}$ at $37^{\circ} \mathrm{C}$ and decolorized by destaining solution (5\% ethanol and $10 \%$ acetic acid glacial) overnight. For Western blotting, the gels were electrotransferred onto a polyvinylidene difluoride membrane (Millipore, Billerica, MA). The membranes were blocked for $1 \mathrm{~h}$ in Tris-buffered saline containing $0.05 \%$ Tween 20 (TBST) and $5 \%$ skimmed milk followed by probing overnight at $4^{\circ} \mathrm{C}$ with primary and secondary antibodies. Mouse anti-His monoclonal antibody, mouse anti-S1 monoclonal antibody 6B4/6G1/1C11 strain, and PEDV-positive serum were used as primary antibodies (dilution 1:2000), and HRP-labeled goat anti-mouse IgG antibody and HRP-labeled goat anti-pig IgG antibody were used as secondary antibodies (dilution 1:2000). The results were visualized using West Pico chemiluminescent substrate (Thermo, Marina, CA) under the conditions recommended by the manufacturer. Images were captured in a Gel 3100 chemiluminescent imaging system (Sagecreation, Beijing, China).

\subsection{PEDV indirect fluorescent antibody (IFA) test}

PEDV indirect fluorescent antibody test methods were described in detail in previous study[25]. Briefly, PEDV-infected cells were fixed at $16 \mathrm{hr}$ after PEDV infection in $4 \%$ paraformaldehyde at $4^{\circ} \mathrm{C}$ overnight and then washed with $1 \times$ PBS $(0.01 \mathrm{M} \mathrm{pH} 7.2)$. Fixed cells were permeabilized with $0.2 \%$ Triton $\mathrm{X}-100$ at room temperature for $15 \mathrm{~min}$ and blocked with PBS containing 10\% BSA for one hour. The samples were reacted with different pig sera as the primary antibodies (dilution 1:200) at $37{ }^{\circ} \mathrm{C}$ for one hour and then labeled with Alexa Fluor 488 goat anti-pig IgA antibody as the secondary antibody (dilution 1:1000) at 37 ${ }^{\circ} \mathrm{C}$ for $1 \mathrm{~h}$. Each serum was tested by IFA three times, and positive and negative serum controls were included throughout the experiment. Cell nuclei were stained with DAPI (Beyotime, China) at a 1:1000 dilution. Images were visualized using a laser confocal microscope IX81-FV1000 (Olympus, Japan). PEDV reference positive and negative sera were used as positive and negative controls.

\subsection{ELISA}

An indirect ELISA was carried out and optimized with porcine serum samples. ELISA process was described in detail in previous study[25]. Briefly, 96-well microplates were coated overnight at $4^{\circ} \mathrm{C}$ with purified S1 truncated protein diluted in $100 \mathrm{mM}$ carbonate-bicarbonate buffer (A500873, Sangon Bitech, China, pH 9.6). The wells were washed 3 times and blocked with $0.05 \mathrm{~g} / \mathrm{mL}$ sucrose (A502792, Sangon Biotech, China), 0.1\% proclin-300 (48912, Sigma, USA) and $0.02 \mathrm{~g} / \mathrm{mL}$ BSA (C510069, Sangon Biotech, China) in $10 \mathrm{mM} \mathrm{PBS}$ at $37^{\circ} \mathrm{C}$. After three washes with PBST (0.01 M pH 7.2 with $0.5 \%$ Tween-20), $100 \mu \mathrm{L}$ of serum from ZJ15XS0101-infected pigs diluted in PBST-5\% milk was added to each well and incubated at $37^{\circ} \mathrm{C}$. After washing, the reaction mixture was incubated with HRP-labeled goat anti-pig IgA antibody (dilution, 1:1500). After incubation and subsequent washing, the color was developed by the addition of $100 \mu \mathrm{L}$ of substrate solution consisting of tetramethylbenzidine chromogenic substrate (Sigma-Aldrich) and stopped by adding $50 \mu \mathrm{L}$ of $2 \mathrm{M} \mathrm{H}_{2} \mathrm{SO}_{4}$. The optical densities at $450 \mathrm{~nm}\left(\mathrm{OD}_{450 \mathrm{~nm}}\right)$ were measured in 
a microplate reader (BioTek, USA). Each serum sample was tested in triplicate, each well was read twice, and the cutoff point of a positive sample was set to be at least twice the negative sample at any dilution. Different antigen concentrations, serum dilutions and incubation times of each step were tested by checkboard assays to optimize the ELISA method.

\subsection{SNT}

The pig serum samples were inactivated at $56^{\circ} \mathrm{C}$ for 30 min and diluted by a factor of 2 , which began at $1: 5$. The virus at $10^{4} \mathrm{TCID}_{50} / \mathrm{mL}$ with diluted serum in the same volume was mixed and incubated at $37^{\circ} \mathrm{C}$ for 2 h. Vero-E6 cells were inoculated into 96-well plates and cultured to $90-95 \%$ confluency. Then, the medium was replaced with neutralized virus. A total of 4 parallel wells were set for each serum dilution sample. Serum and virus were used as the negative and positive controls, respectively. Viruses were removed after $2 \mathrm{~h}$ of absorption. The cells were washed twice with Hank's solution before maintenance medium (DMEM with $8 \mu \mathrm{g} / \mathrm{mL}$ trypsin) was added. The CPE was determined 3 days post infection. The serum neutralization titer was calculated according to the Reed-Muench method.

\subsection{Statistical analysis}

GraphPad Prism (GraphPad Software, USA) are used to analyze the data. All results are presented as the mean \pm SE of triplicate experiments. Statistical significance was tested by one-way analysis of variance (one-way ANOVA)[26].

\section{Results}

\subsection{Eukaryotic expression of $S 1$ truncated protein by the Bac-to-Bac system}

Previously, we established an S1-ELISA method against PEDV IgG [25]. Based on this method, we replaced IgG antibody with IgA antibody in secondary antibody incubation so that we established the S1ELISA method against PEDV IgA. To further improve the specificity of the ELISA detection method, we designed an optimization strategy with truncated S1 protein (Fig. 1A\&B). The recombinant bacmids containing S1T1, S1T2 and S1T3 were successfully constructed and identified by plasmid double digestion identification with target fragments of 4827 bp (pFASTBac plasmid), 924 bp (S1T1), 927 bp (S1T2) and 894 bp (S1T3). We also identified the recombinant bacmids by PCR using M13 primers and specific primers with the expected products (Fig. 1C\&D). The recombinant baculovirus was generated successfully. Cytopathic effects (CPE), such as cell swelling, were observed in infected cells (Fig. 2). Baculovirus infections were also identified by immunofluoresence using a tag antibody (Fig. 3).

Recombinant S1 truncated proteins were identified by SDS-PAGE (Fig. 4A) with Coomassie blue staining and western blotting analysis using a His-tagged antibody (Fig. 4B). We also tested the reactivities of the S1 monoclonal antibody and the recombinant truncated proteins. Monoclonal antibodies 6B4, 6G1 and $1 \mathrm{C11}$ can identify S1T1, S1T2, and S1T3, respectively (Fig. 4C-4E). PEDV-positive serum was also reactive with these truncated proteins (Fig. 4F). 


\subsection{Development of ELISA based on recombinant S1 truncated protein}

The antigen coating conditions were optimized under different temperatures and times (data not shown). The optimal working concentration of antigen coating and the appropriate dilutions of sera were confirmed to be $10 \mu \mathrm{g} / \mathrm{mL}$ and 1:50 for all three truncated proteins, which were determined by checkerboard assays using serial dilutions of antigens and sera (Fig. 5). The conditions and times of antigen coating, antibody incubation and substance reaction were optimized in a previous study[25]. The S1/1S1T1/S1T2/S1T3-ELISA against IgA showed no cross reactivities with CSFV-, PCV2-, PRRSV- and TGEV-positive serum (data not shown).

\subsection{ROC analysis of ELISA}

In 213 pig serum samples, 75 (35.21\%) and 138 (64.79\%) were determined to be PEDV IgA positive and negative, respectively. Typical IgA IFA results are shown in Fig. 6 . Taking the IFA results as gold standard, the sensitivity and specificity of ELISA were analyzed by the ROC curves. The ROC analysis data are shown in Fig. 7A and Table 2 with the cutoff value, sensitivity and specificity, the area under the curve (AUC), and the 95\% confidence interval of S1/S1T1/S1T2/S1T3-ELISA. The S1/S1T1/S1T2/S1T3-ELISA against IgA results presented 78/59/65/70 positive samples and 135/154/138/143 negative samples, containing 9/4/10/13 false-positive and 6/20/10/18 false-negative samples, respectively (Fig. 7B). Together, these results indicate that the S1-ELISA and S1T2-ELISA against IgA presented high relative sensitivity and relative specificity.

Table 2

ROC results of PEDV S1/S1T1/S1T2/S1T3 ELISA

\begin{tabular}{|lllll|}
\hline & S1 & T1 & T2 & T3 \\
\hline cut-off & 0.4129 & 0.7102 & 0.3311 & 0.3707 \\
\hline sensitivity & 93.48 & 97.1 & 92.75 & 90.58 \\
\hline Specificity & 92 & 73.33 & 86.67 & 76 \\
\hline AUC & $0.9683 \pm 0.0110$ & $0.9001 \pm 0.0248$ & $0.9392 \pm 0.020$ & $0.9044 \pm 0.0234$ \\
\hline $\begin{array}{l}\text { 95\% confidence } \\
\text { interval }\end{array}$ & 0.9449 to & 0.8515 to & 0.8999 to & 0.8585 to \\
\hline P value & 0.9916 & 0.9487 & 0.9785 & 0.9504 \\
\hline
\end{tabular}

\subsection{IgA antibody by ELISA could reflect the neutralization antibody titer}

We tested 109 samples of pig serum collected from local farms in Zhejiang Province. Serum was divided into two groups: a high group with SNT $\geq 40$ and a low group with SNT < 40 (Fig. 8A). 
A total of 109 pig serum samples were tested for the presence of PEDV IgA by S1/S1T1/S1T2/S1T3ELISA and tested for the neutralization antibody titer. The ELISA results showed significant relevance to the serum neutralization titer (Fig. 8B). The ELISA OD ${ }_{450 \mathrm{~nm}}$ value of serum with a high neutralization antibody titer was significantly higher than that with a low neutralization antibody titer. These results indicate that our ELISA methods based on S1 and its truncated proteins could reflect the neutralization capability of the serum sample.

\section{Discussion}

As a porcine intestinal disease, PED causes very similar clinical symptoms to other intestinal coronavirus diseases, such as TGEV and porcine deltacoronavirus (PDCoV). Therefore, laboratory tests are necessary to confirm the diagnosis. The methods currently available for PEDV detection include virus isolation and identification, immunoelectron microscopy, immunohistochemistry, virus neutralization test, etc. However, the experimental operation is more complicated. Although RT-PCR can achieve rapid detection of PEDV, serological testing can not only achieve rapid detection but also monitor the changes in antibody levels in pigs, which could be used to evaluate the immune effect after vaccination. M. Knuchel et al. used PEDV S protein and $\mathrm{N}$ protein as coating antigens to establish an ELISA detection system[23]. The results showed that S-ELISA is more sensitive than N-ELISA, and antibodies against S protein last longer in the body than $\mathrm{N}$ protein. The good immunogenicity and reactogenicity of the $\mathrm{S}$ protein make it an ideal target protein for PEDV detection. The S1 protein, as a hyper-various mutagenic virulence-related gene-encoded protein, made the existing vaccines unable to provide complete protection against the current PEDV epidemic strains. The current prevention and control of PEDV is still focused on vaccination. Therefore, it is very important to establish an effective method for evaluating the effect of vaccination to speed up the development of new vaccines. Most of the identified epitopes are located in the S1 region[36], and the variability of the S1 sequence is higher than that of the S2 subunit. Therefore, the PEDV S1 protein can be used as an ideal target protein for PEDV diagnosis and vaccine development.

We obtained truncated S1 proteins with good immunogenicity that were close to their natural conformation through the baculovirus expression system. The baculovirus expression system, as a widely used and highly efficient eukaryotic system, can allow the insertion of larger exogenous DNA fragments. The polyhedron or strong p10 promoter contained in the system can induce high expression of the target gene. Compared with prokaryotic expression, this system can modify and process translated proteins, such as glycosylation, acylation, phosphorylation, peptide cleavage and disulfide bond formation. The expressed protein is closer to natural products and has high protein activity and immunogenicity. Credit to signal peptide genetic modification, it is easy to obtain proteins from the culture supernatants, which were of high purity and large amounts for ELISA antigen coating.

The ROC curve is a tool to evaluate the diagnostic value of the established detection method by calculating the area under the curve (AUC). The Youden index is often used as the cut-off for negative and positive sera. The larger the area under the curve, the higher the Youden index, and the more reliable the established detection method. We used IFA results as the standard to analyze the established indirect 
ELISA detection method for anti-PEDV IgA antibody based on S1 and its truncated protein. IgA antibody ELISA showed a significant $P$ value of ROC analysis and large AUCs. The ROC areas under the curve were $0.968,0.905,0.944$ and 0.909 for IgA ELISA based on S1, S1T1, S1T2 and S1T3, respectively. Among them, the established S1-ELISA and S1T2-ELISA methods exhibited high sensitivity and specificity of $93.5 \%$ and $92.0 \%$ and $92.8 \%$ and $86.7 \%$, respectively. The $\mathrm{OD}_{450 \mathrm{~nm}}$ values of S1-ELISA and S1T2-ELISA against IgA had a significant correlation with a correlation coefficient of 0.9134 . In addition, the $\mathrm{OD}_{450 \mathrm{~nm}}$ values determined by S1-ELISA and S1T2-ELISA against IgA in serum with a high neutralization titer were significantly higher than those with a low neutralization titer. It is suggested that S1-ELISA and S1T2ELISA against IgA could also be used as candidate systems for detecting the titer of anti-PEDV IgA antibodies in serum. These established ELISA detection methods could reflect the neutralization of natural infection and vaccinated pigs to a certain extent of active neutralization antibody levels.

PEDV mainly destroys intestinal epithelial cells, causing diarrhea, dehydration and mass death in suckling piglets. In vaccinated and infected pigs, IgG and IgA collectively contribute to the neutralizing antibody titer. The specific IgA antibody taken from colostrum is the main antibody for suckling piglets to resist PEDV infection. Mucosal IgA has a higher ability to neutralize viruses in the intestine[33]. Vaccination is widely used for the prevention and control of PEDV in China, and IgA detection in sow serum could be reflective to some extent of the neutralization titer in colostrum. The serum IgA antibody ELISA detection method based on the $S$ protein can be used for PEDV infection detection and vaccine immunity evaluation. In addition, it can also detect the IgA antibody in colostrum to predict the risk of diarrhea in piglets. The antibody level detected from these methods could provide a basis for effective prevention and control measures of PEDV, such as supplementary immunization for sows with low antibody levels.

Taken together, the established S1-ELISA and S1T2-ELISA against PEDV IgA could serve as candidate methods for detecting the anti-PEDV IgA antibody level in serum, and the antibody level determined by these ELISA methods could reflect the level of neutralization capability in naturally infected and vaccinated pigs.

\section{Declarations}

\section{Ethics approval and consent to participate}

The experimental materials and procedures involving animals in this study were approved by by the Animal Care and Use Committee of Zhejiang University (Approval No. ZJU20170360). All methods were performed in accordance with the relevant guidelines and regulations. The study was carried out in compliance with the ARRIVE guidelines.

\section{Acknowledgements}

We thanked the Shared Experimental Platform for Core Instruments in College of Animal Science, Zhejiang University for assistance with analysis of laser confocal microscopy imaging. 


\section{Funding}

This work was financially supported by grants from the Analysis and Measurement Foundation of Zhejiang Province (LGC20C180001), the "Pioneer" and "Leading Goose" R\&D Program of Zhejiang (2022C02031), the Zhejiang Provincial Key R\&D Program of China (2021C02050, 2021C02049), the science and technology innovation leading talents project of "High-level Talents Special Support Plan" of Zhejiang Province (2021R52041), and the agricultural major technology synergy extension project of Zhejiang Province (2021XTTGXM02-05).

\section{Author Contributions}

Ying Shan wrote the main manuscript text; Ying Shan, Weihuan Fang and Xiaoliang Li designed the study concept and strategy; Zheng Jingyou, Xiaohan Xu, Chuni Zhang and Xiaojun Huang obtained the S1 truncated protein; Qin Gao and Junyong Mao established and optimized the ELISA methods; Ying Shan and Jidong Xu collected and analyzed the ELISA and neutralization test data; Fushan Shi, Fang He and Min Yue critically revised the manuscript. All authors reviewed the manuscript.

\section{Competing interests}

The authors declare that there are no conflicts of interest.

\section{Availability of data and materials}

The datasets generated during and analyzed during the current study are not publicly available due to lab server breakdown but are available from the corresponding author on reasonable request.

\section{Consent for publication}

Not Applicable.

\section{References}

1. Wood, E.N., An apparently new syndrome of porcine epidemic diarrhoea. Vet Rec, 1977. 100(12): p. 243-4.

2. Pensaert, M.B. and P. de Bouck, A new coronavirus-like particle associated with diarrhea in swine. Arch Virol, 1978. 58(3): p. 243-7.

3. Martelli, P., et al., Epidemic of diarrhoea caused by porcine epidemic diarrhoea virus in Italy. Vet Rec, 2008. 162(10): p. $307-10$.

4. Sun, R.Q., et al., Outbreak of porcine epidemic diarrhea in suckling piglets, China. Emerg Infect Dis, 2012. 18(1): p. 161-3.

5. Zhi, Z., et al., Survey and surveillance of porcine epidemic diarrhea in some provinces in China. China Animal Health Inspection, 2014. 
6. Wang, et al., Porcine epidemic diarrhea in China. Virus Research: An International Journal of Molecular and Cellular Virology, 2016.

7. Li, W., et al., New variants of porcine epidemic diarrhea virus, China, 2011. Emerg Infect Dis, 2012. 18(8): p. 1350-3.

8. Puranaveja, S., et al., Chinese-like strain of porcine epidemic diarrhea virus, Thailand. Emerg Infect Dis, 2009. 15(7): p. 1112-5.

9. Lee, C., Porcine epidemic diarrhea virus: an emerging and re-emerging epizootic swine virus. Virology journal, 2015. 12(1): p. 193.

10. Song, D. and B. Park, Porcine epidemic diarrhoea virus: a comprehensive review of molecular epidemiology, diagnosis, and vaccines. Virus Genes, 2012. 44(2): p. $167-75$.

11. Huang, Y.W., et al., Origin, evolution, and genotyping of emergent porcine epidemic diarrhea virus strains in the United States. MBio, 2013. 4(5): p. e00737-13.

12. Sun, M., et al., Genomic and Epidemiological Characteristics Provide New Insights into the Phylogeographical and Spatiotemporal Spread of Porcine Epidemic Diarrhea Virus in Asia. Journal of Clinical Microbiology, 2015. 53(5): p. 1484-1492.

13. Belouzard, S., et al., Mechanisms of coronavirus cell entry mediated by the viral spike protein. Viruses, 2012. 4(6): p. 1011-33.

14. Park, S.J., et al., Cloning and further sequence analysis of the ORF3 gene of wild- and attenuatedtype porcine epidemic diarrhea viruses. Virus Genes, 2008. 36(1): p. 95-104.

15. de Haan, C.A.M., et al., Cleavage of group 1 coronavirus spike proteins: How furin cleavage is traded off against heparan sulfate binding upon cell culture adaptation. Journal of Virology, 2008. 82(12): p. 6078-6083.

16. Madson, D.M., et al., Pathogenesis of porcine epidemic diarrhea virus isolate (US/lowa/18984/2013) in 3-week-old weaned pigs. Vet Microbiol, 2014. 174(1-2): p. 60 - 8.

17. Song, Y., et al., A Computationally Designed Serological Assay for Porcine Epidemic Diarrhea Virus. Journal of Clinical Microbiology, 2016. 54(8): p. 2039-2046.

18. Song, Y., et al., A Computationally Designed Serological Assay for Porcine Epidemic Diarrhea Virus. J Clin Microbiol, 2016. 54(8): p. 2039-46.

19. Fan, J.H., et al., Development of an enzyme-linked immunosorbent assay for the monitoring and surveillance of antibodies to porcine epidemic diarrhea virus based on a recombinant membrane protein. J Virol Methods, 2015. 225: p. 90 - 4.

20. Gerber, P.F. and T. Opriessnig, Detection of immunoglobulin (Ig) A antibodies against porcine epidemic diarrhea virus (PEDV) in fecal and serum samples. MethodsX, 2015. 2: p. 368 - 73.

21. Carvajal, A., et al., Evaluation of a Blocking Elisa Using Monoclonal-Antibodies for the Detection of Porcine Epidemic Diarrhea Virus and Its Antibodies. Journal of Veterinary Diagnostic Investigation, 1995. 7(1): p. 60-64. 
22. Lindstrom, P. and O. Wager, IgG autoantibody to human serum albumin studied by the ELISAtechnique. Scand J Immunol, 1978. 7(5): p. 419 - 25.

23. Knuchel, M., et al., An ELISA for detection of antibodies against porcine epidemic diarrhoea virus (PEDV) based on the specific solubility of the viral surface glycoprotein. Vet Microbiol, 1992. 32(2): p. $117-34$.

24. Woof, J.M. and M.A. Kerr, The function of immunoglobulin A in immunity. The Journal of Pathology, 2006. 208(2): p. 270-282.

25. Shan, Y., et al., Development and application of an indirect enzyme-linked immunosorbent assay using recombinant $\mathrm{S} 1$ for serological testing of porcine epidemic diarrhea virus. Canadian Journal of Microbiology, 2019.

26. Bewick, V., L. Cheek, and J. Ball, Statistics review 9: One-way analysis of variance. Critical Care, 2004. 8(2).

\section{Figures}

A S

(1387 aa)

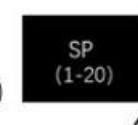

S1
$(21-788)$

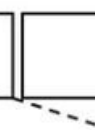

S2

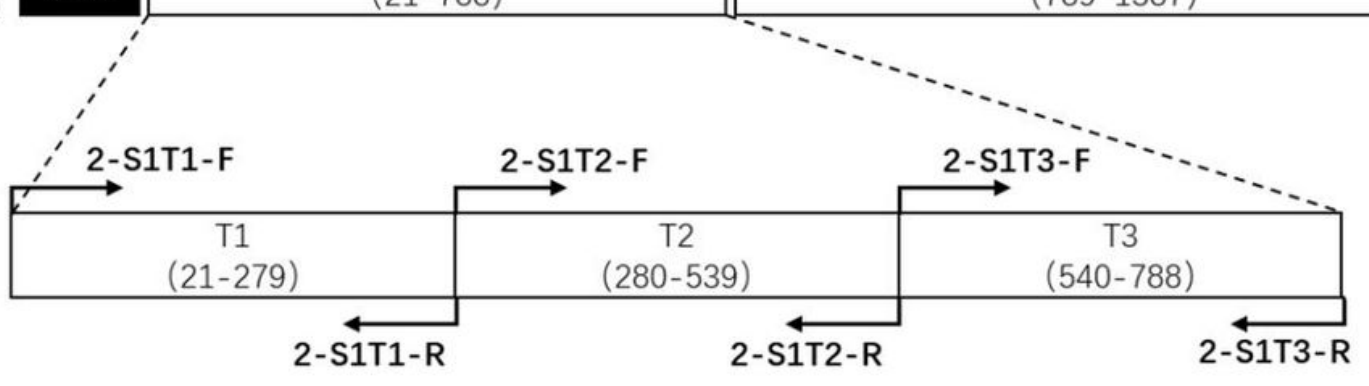

B

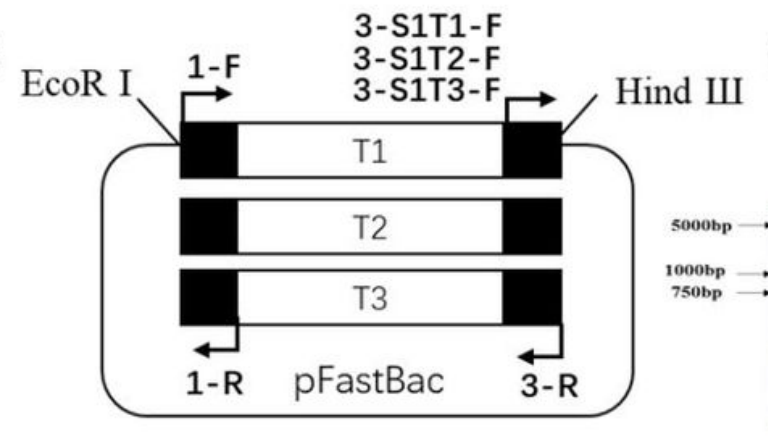

C

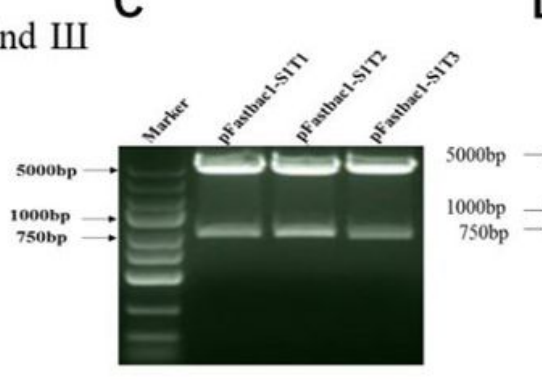

$\mathrm{D}$

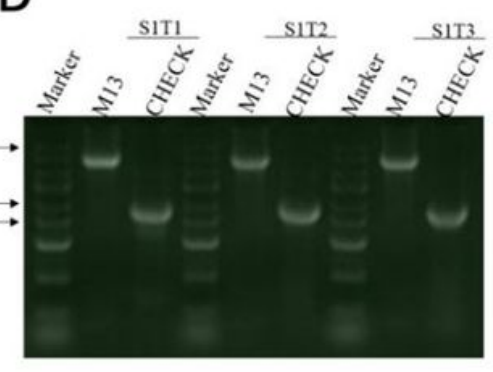

\section{Figure 1}

The construction of S1T1, S1T2 and S1T3 recombinant balculovirus. A. Division strategy of PEDV S1 gene into three segments as S1T1, S1T2 and S1T3. B. Construction strategy of S1T1, S1T2 and S1T3 recombinant balculovirus plasmids. C. Identification of recombinant balculovirus plasmids by double digestion identification with target fragments of 4827 bp (pFASTBac plasmid), 924 bp (S1T1), 927 bp 
(S1T2) and $894 \mathrm{bp}$ (S1T3). D. Identification of the recombinant bacmids by PCR with expected products respectively.
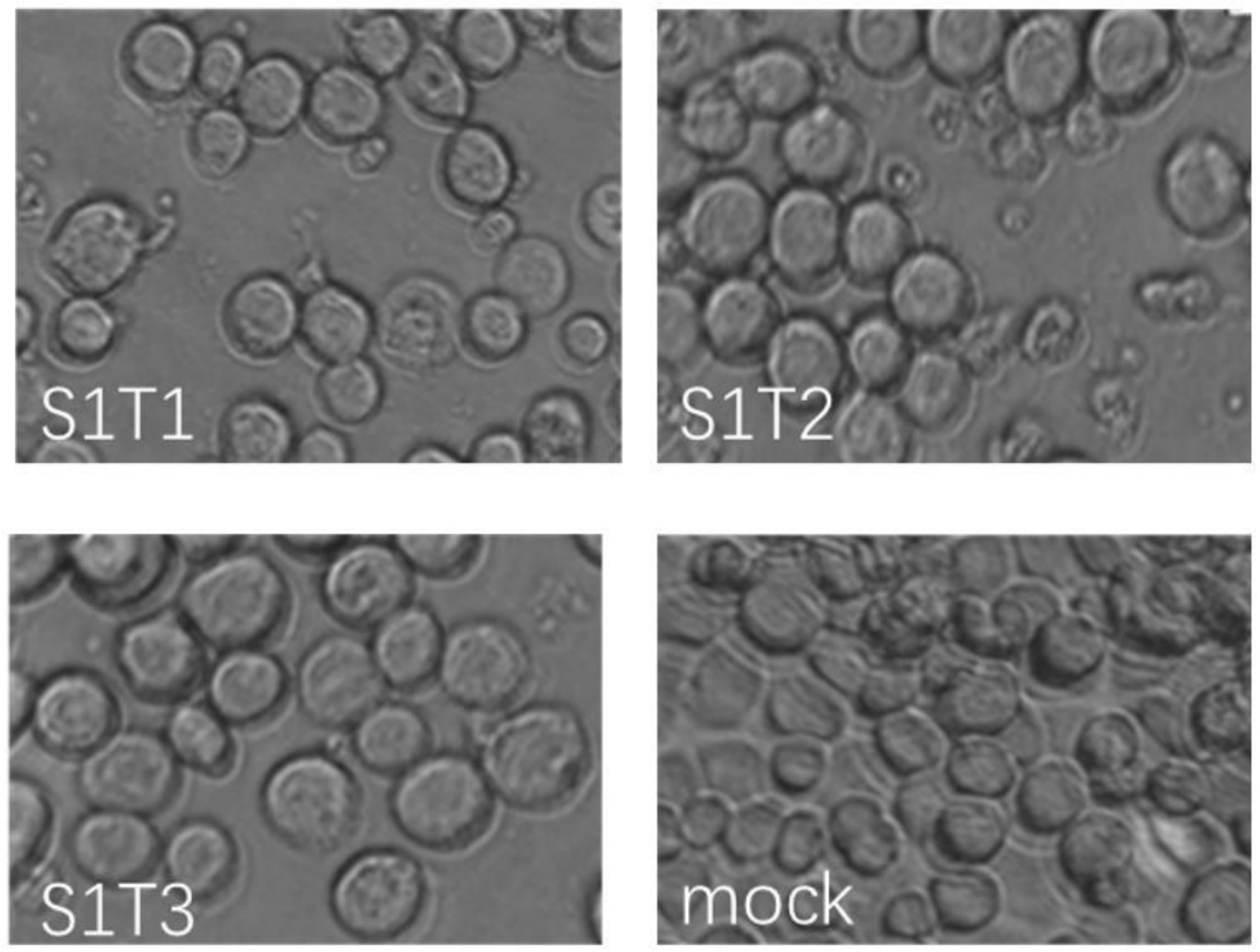

\section{Figure 2}

Identification of the recombinant baculovirus infection by cytopathic effects. The recombinant baculovirus were generated successfully. Cytopathic effects (CPE) such as cell swelling was observed in infected cells. 


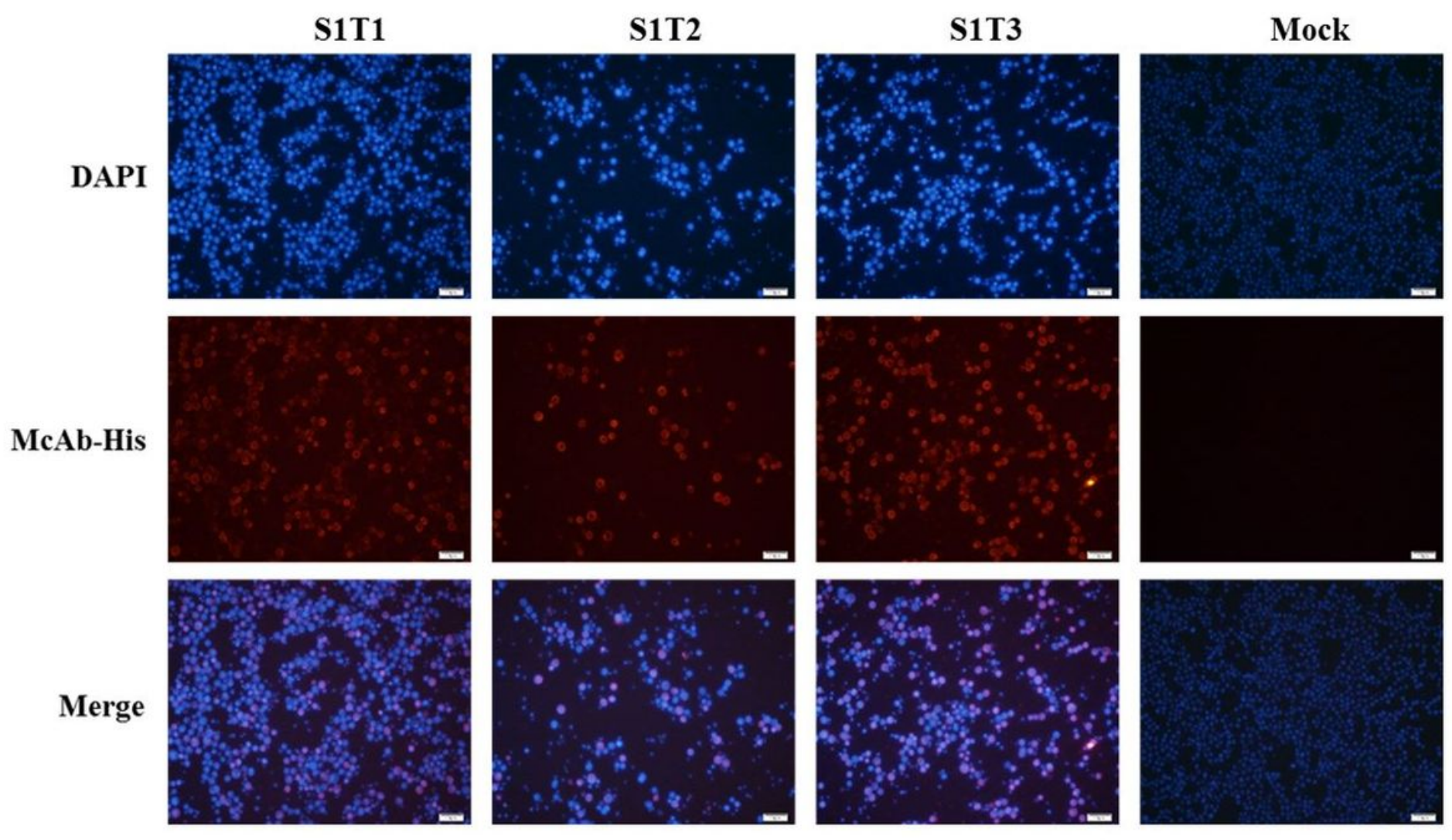

Figure 3

Identidication of the recombinant baculovirus infection by immunofluoresence assay. Baculovirus infections were identified by immunofluoresence using his tag monoclonal antibody. Positive cells (red) with recombinant S1T1/S1T2/S1T3 expression were observed in infected cells.
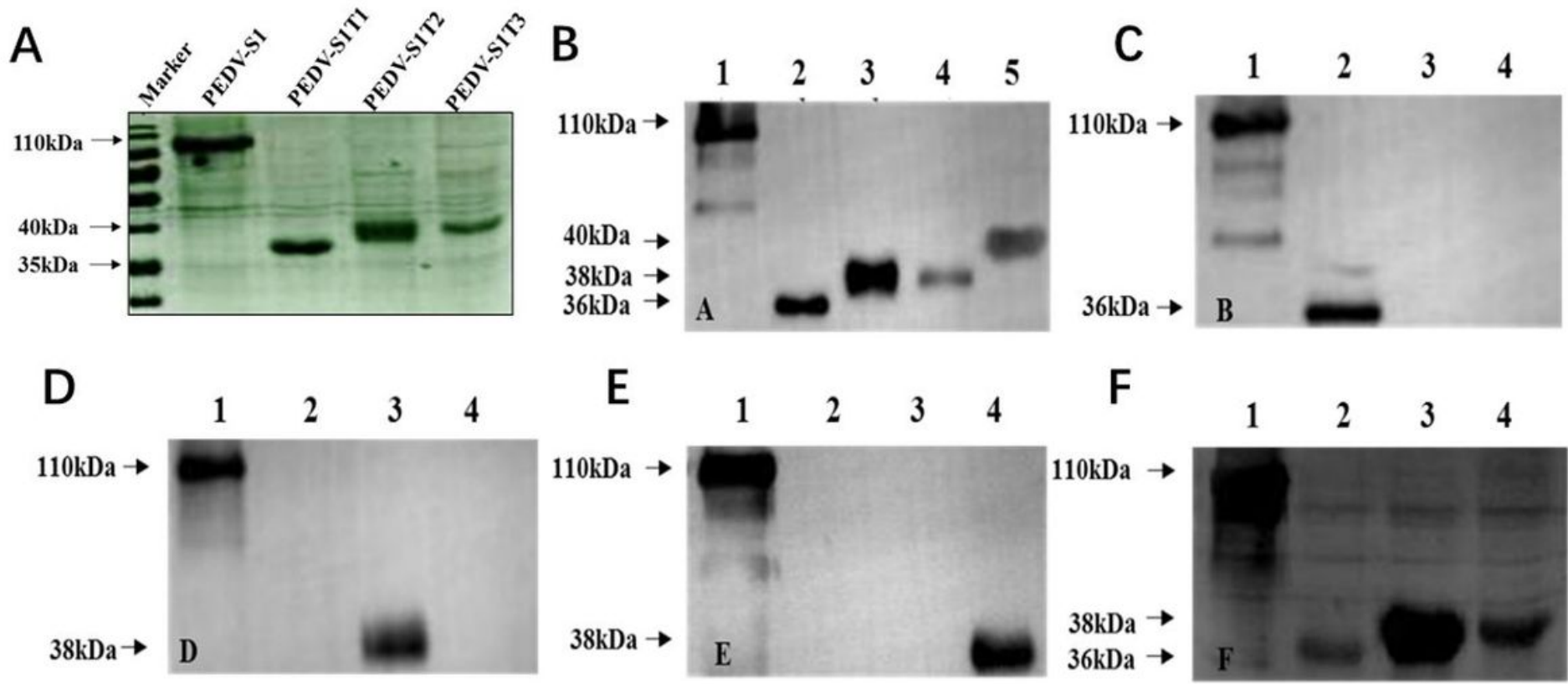
Figure 4

Identification of recombinant S1 truncated protein expression. A. Recombinant S1 truncated proteins were identified by SDS-PAGE with Coomassie blue staining; B. Recombinant S1 truncated proteins were identified by western blotting analysis using his-tag antibody. C-F. S1 antibody reactivity with the recombinant S1 truncated protein. Monoclonal antibody 6B4, 6G1, 1C11 can identify S1T1, S1T2, S1T3, respectively. PEDV positive serum could identify all of these truncated proteins. (Lane1: PEDV-S1; Lane2: PEDV-S1T1; Lane3: PEDV-S1T2; Lane4: PEDV-S1T3; Lane5: His tag positive control; A: SDS-PAGE with Coomassie blue staining; B: anti-His McAb; C: anti-PEDV S1 McAb-6B4; D: anti-PEDV S1 McAb-6G1; E: anti-PEDV S1 McAb-1C11; F: PEDV positive pig serum
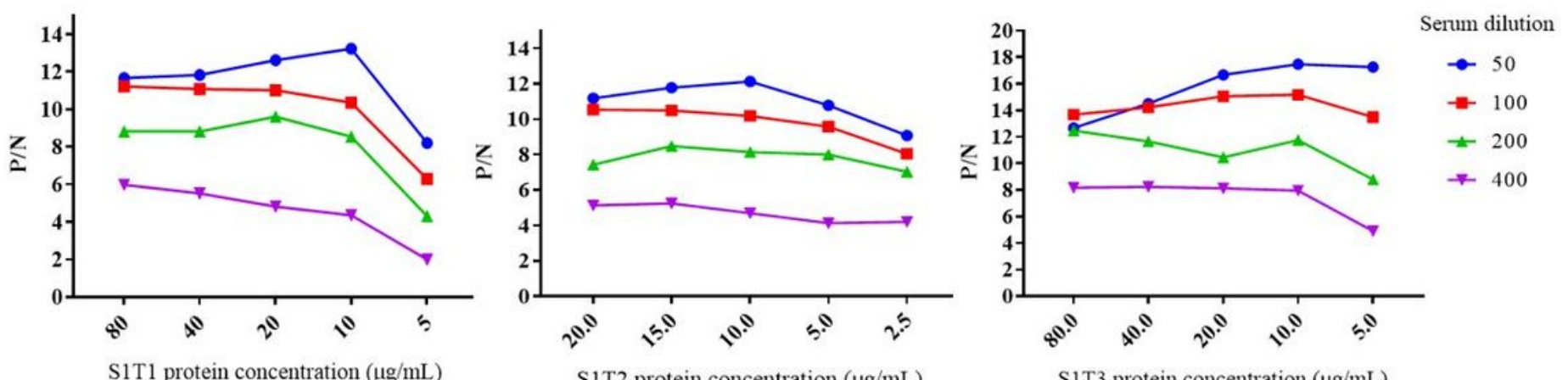

S1T2 protein concentration $(\mu \mathrm{g} / \mathrm{mL})$

S1T3 protein concentration $(\mu \mathrm{g} / \mathrm{mL})$

\section{Figure 5}

Optimization of coating antigen concentration and serum dilution of truncated S1 ELISA. The optimal working concentration of antigen coating and the appropriate dilutions of sera were confirmed to be 10 $\mu \mathrm{g} / \mathrm{mL}$ and 1:50 for all the three truncated proteins. 


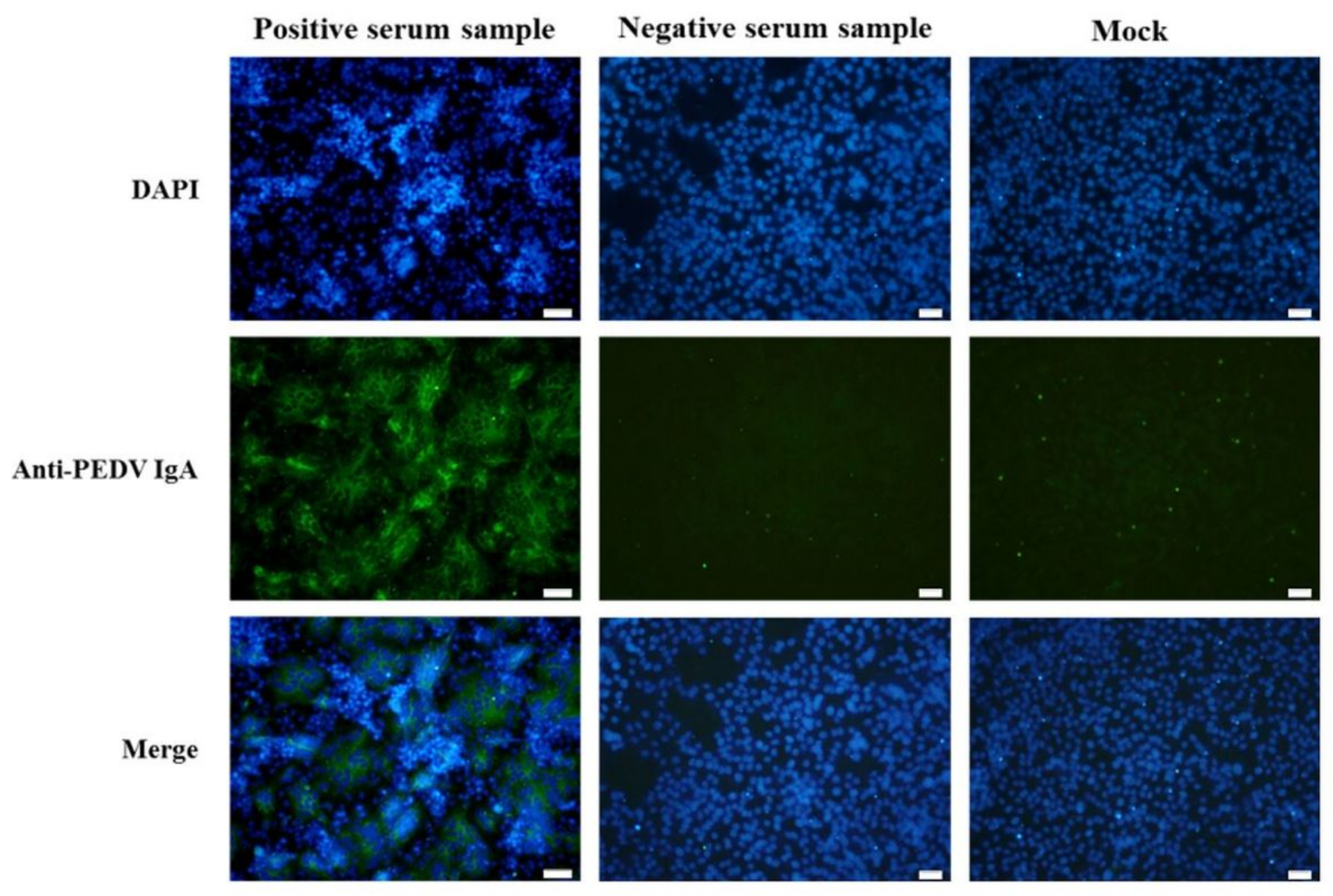

Figure 6

Representative results of immunofluorescence. Out of 213 pig serum samples from local farms, 75 (35.21\%) and 138 (64.79\%) were shown to be PEDV IgA positive and negative, respectively. 
A
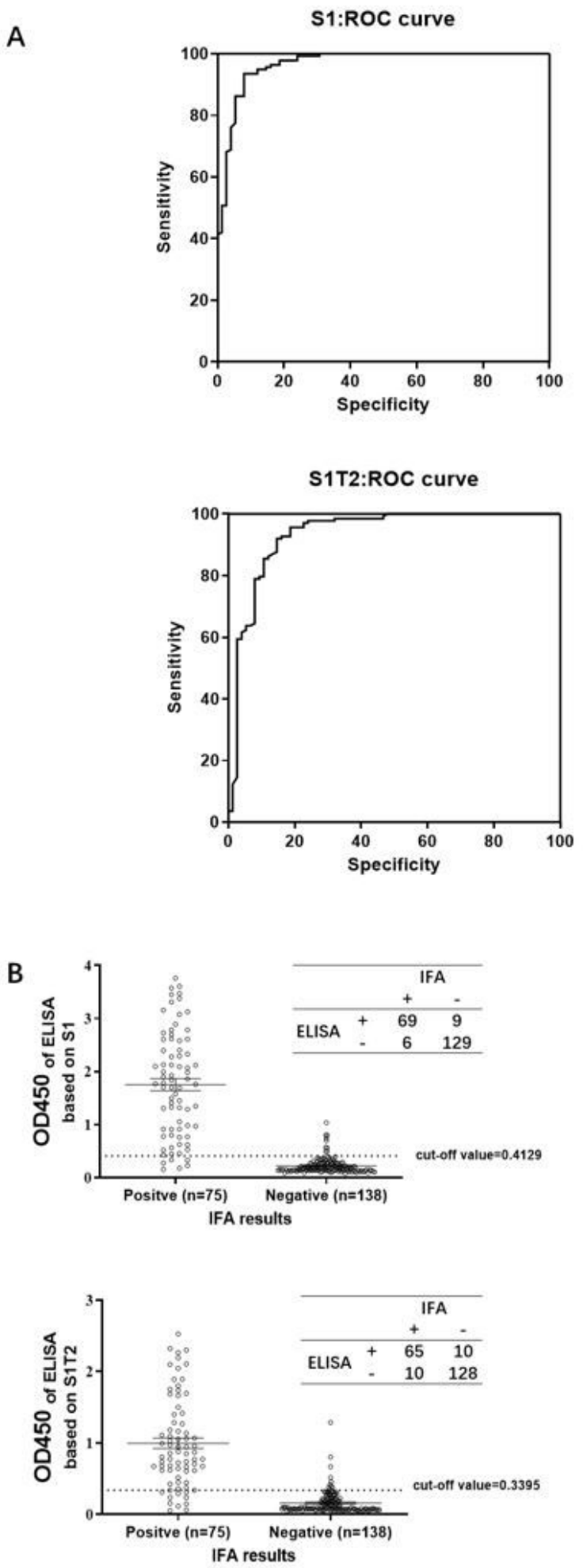
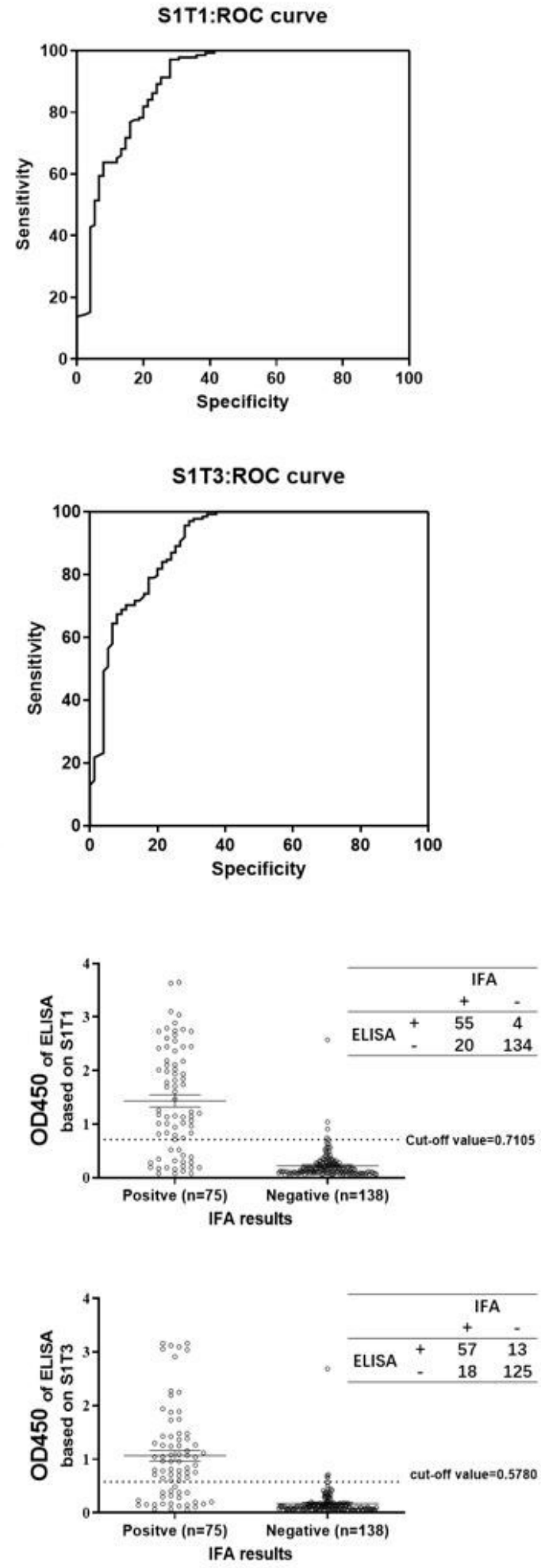

Figure 7

ROC Curve of truncated S1 ELISA taking IFA results as standard. A. A total of 213 pig serum samples were tested for the presence of anti-PEDV IgA by S1/S1T1/S1T2/S1T3-ELISA. The cutoff value of S1/S1T1/S1T2/S1T3-ELISA was $0.4129,0.7102,0.3311$ and 0.3707 , respectively; the sensitivity (\%)/specificity (\%) of S1/S1T1/S1T2/S1T3-ELISA was 93.48/92, 97.1/73.33, 92.75/86.67, 90.58/76.00, respectively; the area under curve (AUC) of S1/S1T1/S1T2/S1T3-ELISA was 0.9683 0.0110 , 
$0.9001 \pm 0.0248,0.9392 \pm 0.020$ and $0.9044 \pm 0.0234$, respectively. B. The S1/S1T1/S1T2/S1T3-ELISA against IgA results presented 78/59/65/70 positive samples and 135/154/138/143 negative samples, containing 9/4/10/13 false-positive and 6/20/10/18 false-negative samples, respectively.

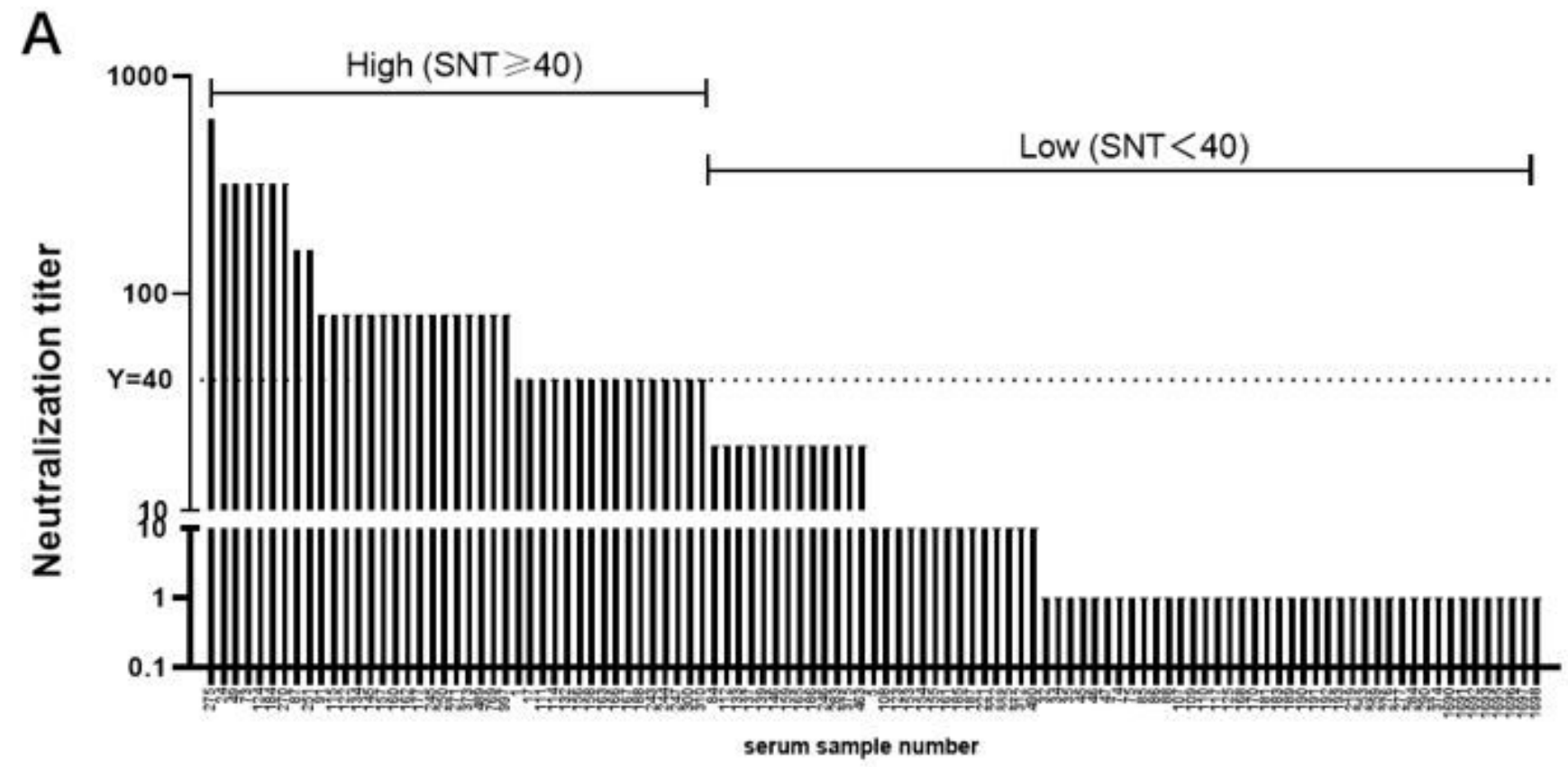

B

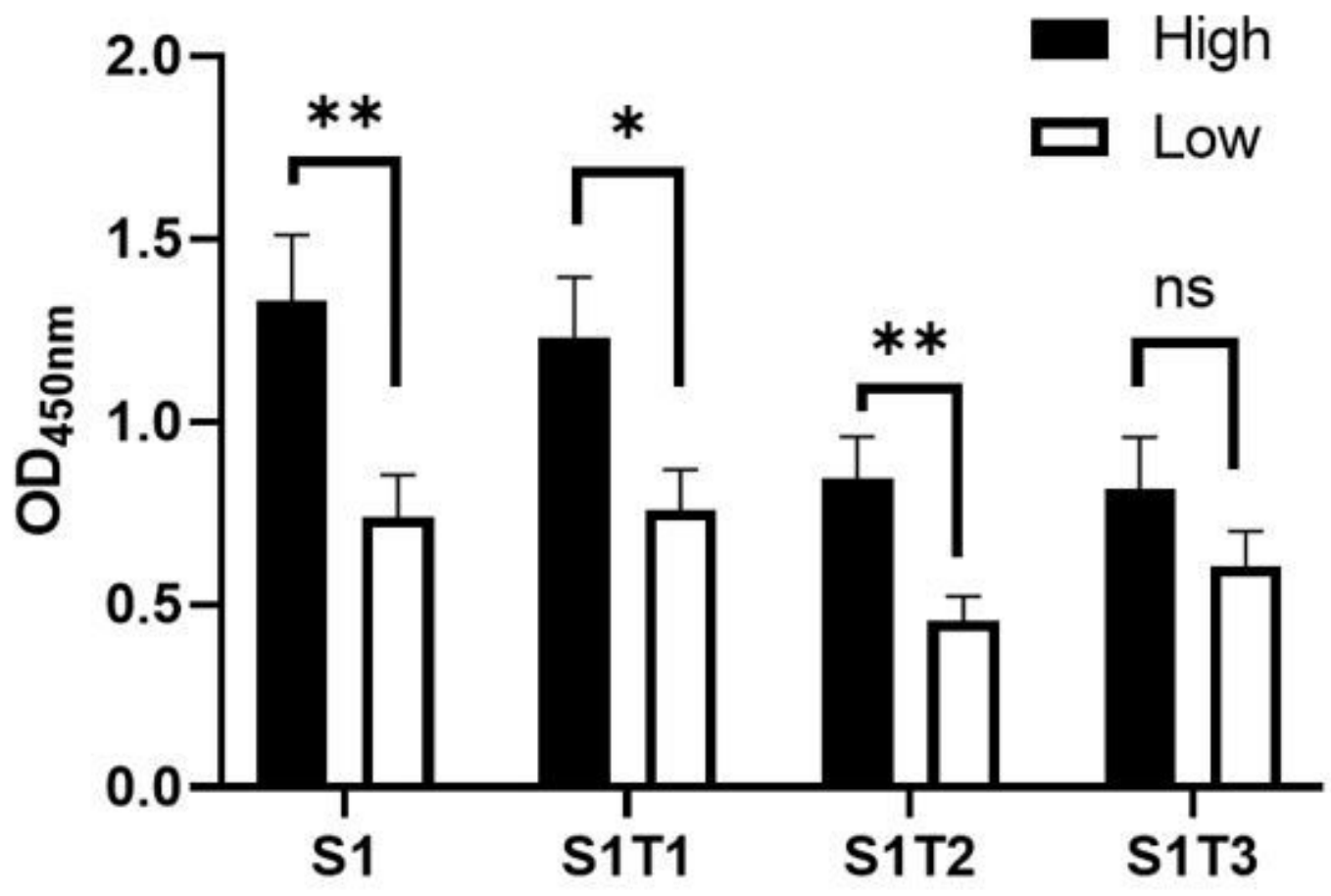

Figure 8

Relevance between S1T1/S1T2/S1T3-ELISA value with serum neutralization titer. A. Serum group was determinated by serum neutralization test (SNT), high group was $S N T \geq 40$ and low group was $S N T<40 ;$ B. the relevance between the results of S1/S1T1/S1T2/S1T3-ELISA against PEDV IgA with serum 
neutralization titer. A total of 109 pig serum samples were tested for the presence of anti-PEDV IgG and IgA by S1T1/S1T2/S1T3-ELISA and tested for the neutralization antibody titer. The IgA ELISA OD $450 \mathrm{~nm}$ value of serum with high neutralization antibody titer was significantly higher than that with low neutralization antibody titer, while there was no significant correlation between IgG ELISA OD $450 \mathrm{~nm}$ value of neutralization antibody titer. These results indicate that IgA-ELISA based on the S1 truncated proteins could reflect the neutralization capability of the serum sample.

\section{Supplementary Files}

This is a list of supplementary files associated with this preprint. Click to download.

- SDSpage.jpg 